\title{
EURECOM
}

Institut Eurécom

Department of Mobile Communications

2229, route des Crêtes

B.P. 193

06904 Sophia-Antipolis

FRANCE

Research Report RR-06-168

\section{Mobility Models for Vehicular Ad Hoc Networks: A Survey and Taxonomy}

March 5 $5^{\text {th }}, 2006$

Last update March $26^{\text {th }}, 2007$

Jérôme Härri, Fethi Filali and Christian Bonnet

Tel : (+33) 493008100

Fax : (+33) 493008200

Email : $\{$ Jerome.Haerri,Fethi.Filali,Christian.Bonnet $\} @$ eurecom.fr

\footnotetext{
${ }^{1}$ Institut Eur'ecom's research is partially supported by its industrial members: BMW Group Research \& Technology - BMW Group Company, Bouygues T’el’ecom, Cisco Systems, France T’el'ecom, Hitachi Europe, SFR, Sharp, STMicroelectronics, Swisscom, Thales.
} 



\title{
Mobility Models for Vehicular Ad Hoc Networks: A Survey and Taxonomy
}

\author{
Jérôme Härri, Fethi Filali and Christian Bonnet
}

\begin{abstract}
Vehicular Ad-hoc Networks (VANETs) have been recently attracting an increasing attention from both research and industry communities. One of the challenges posed by the study of VANETs is the definition of a generic mobility model providing an accurate, realistic vehicular mobility description at both macroscopic and microscopic levels. Today, most mobility models for vehicular studies only consider a limited macro-mobility, involving restricted vehicles movements, while little or no attention is paid to micro-mobility and its interaction with the macro-mobility counterpart. On the other hand, the research community cannot have access to realistic traffic generator which have not been designed to collaborate with network simulators. In this paper, we first introduce a classification of existing methods for the generation of vehicular mobility models, then we describe the various approaches used by the community for realistic VANET simulations. Finally, we provide an overview and comparison of a large range of mobility models proposed for vehicular ad hoc networks.
\end{abstract}

\section{Index Terms}

Survey, Taxonomy, Classification, Mobility Models, Traffic generator, Vehicle Ad Hoc Networks. 



\section{Contents}

1 Introduction 1

2 A Framework for Realistic Vehicular Mobility Models 2

3 Generating Mobility Models for Vehicular Networks 3

3.1 Synthetic Models . . . . . . . . . . . . . . . . . . . 4

3.2 Survey-based Models . . . . . . . . . . . . . . . . 5

3.3 Trace-based Models . . . . . . . . . . . . . . 5

3.4 Traffi c Simulator-based Models . . . . . . . . . . . . . . . . . 7

4 Mobility Models and Network Simulators: The Non-Speaking talking to the Deaf

5 A Taxonomy of existing Synthetic VANETs Mobility Models 12

5.1 Taxonomy Criteria . . . . . . . . . . . . . 12

5.1 .1 Macro-mobility Criteria $\ldots \ldots \ldots . \ldots 12$

5.1 .2 Micro-mobility Criteria . . . . . . . . . . . . . . . . 14

5.1.3 Simulator Related Criteria . . . . . . . . . . . . . . 16

5.2 Taxonomy of Synthetic Vehicular Models . . . . . . . . . . 17

6 Conclusion 25 


\section{List of Figures}

1 Proposed concept map of mobility model generation for inter-vehicle

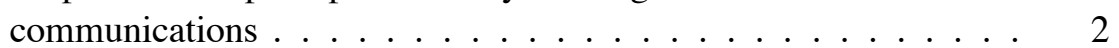

2 Classifi cation of Vehicular Mobility Models . . . . . . . . . . 4

3 Classifi cation of Synthetic Mobility Models . . . . . . . . . . 4

4 Interaction between Network and Traffi c Simulators: The Isolated Case . . . . . . . . . . . . . . . . 8

5 Interaction between Network and Traffi c Simulators: The Integrated Case . . . . . . . . . . . . . . . . . 9

6 Interaction between Network and Traffi c Simulators: The Federated Case . . . . . . . . . . . . . . . . . . 10

7 Road topologies examples . . . . . . . . . . . . . . . 13

8 Example of Attraction Points on a User-defi ned graph . . . . . . . 14

9 Activity-based Sequence between the attraction points in Fig. 8 . . 14

10 General Schema for Car Following Models . . . . . . . . . 15

11 Intersection management in IDM_IM and IDM_LC . . . . . . . 16 


\section{Introduction}

Vehicular Ad-hoc Networks (VANETs) represent a rapidly emerging, particularly challenging class of Mobile Ad Hoc Networks (MANETs). VANETs are distributed, self-organizing communication networks built up by moving vehicles, and are thus characterized by a very high node mobility and limited degrees of freedom in the mobility patterns. Such particular features often make standard networking protocols ineffi cient or unusable in VANETs, whence the growing effort in the development of communication protocols which are specific to vehicular networks.

While it is crucial to test and evaluate protocol implementations in a real testbed environment, simulation is widely considered as a fi rst step in the development of protocols as well as in the validation and refi nement of analytical models for VANETs.

One of the critical aspects when simulating VANETs is the employment of mobility models that reflect as closely as possible the real behavior of vehicular traffi c. This notwithstanding, using simple random-pattern, graph-constrained mobility models is a common practice among researchers working on VANETs. There is no need to say that such models cannot describe vehicular mobility in a realistic way, since they ignore the peculiar aspects of vehicular traffic, such as cars acceleration and deceleration in presence of nearby vehicles, queuing at roads intersections, traffi $\mathrm{c}$ bursts caused by traffi $\mathrm{c}$ lights, and traffi $\mathrm{c}$ congestion or traffi $\mathrm{c}$ jams. All these situations greatly affect the network performance, since they act on network connectivity, and this makes the adoption of a realistic mobility model fundamental when studying VANETs.

In this paper, we describe how vehicular mobility models may be classifi ed in four classes according to the methods used to generate them. Then, we illustrate the particular relationship between network simulators and traffi $\mathrm{c}$ generators used for civil and transportation studies. Finally, we investigate the degree of realism of the different mobility models freely available to the research community on vehicular ad hoc networks. Realism is based on a framework related to realistic vehicular behavior and urban confi gurations. According to it, we give a broad view of the state-of-the-art mobility models adapted for VANETs. To the best of our knowledge, this is the fi st work that provides a detailed survey and comparison of mobility models for vehicular ad hoc networks.

The rest of this paper is organized as follows. Section 2 describes the framework related to realistic vehicular motions, while Section 3 provides a description of the process of generating vehicular mobility models. Then, in Section 4, we cover the relationship between network and traffi c simulators, and in Section 5, we propose a detailed survey and a taxonomy of mobility models available to the vehicular networking community. Finally, in Section 6, we conclude this survey, and provide some hints on future orientation of realistic vehicular mobility models. 


\section{A Framework for Realistic Vehicular Mobility Models}

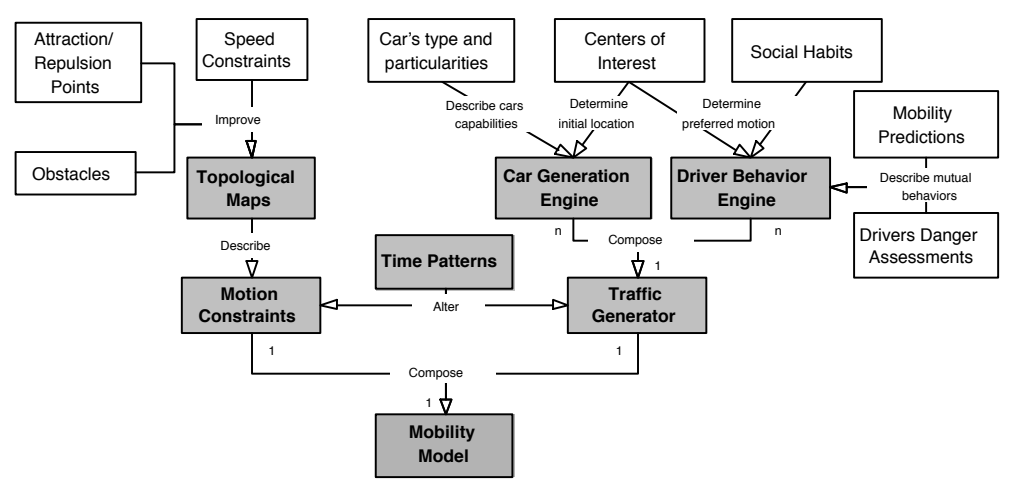

Figure 1: Proposed concept map of mobility model generation for inter-vehicle communications

In the literature, vehicular mobility models are usually classifi ed as either microscopic or macroscopic. When focusing on a macroscopic point of view, motion constraints such as roads, streets, crossroads, and traffi c lights are considered. Also, the generation of vehicular traffi c such as traffi c density, traffi c flows, and initial vehicle distributions are defi ned. The microscopic approach, instead, focuses on the movement of each individual vehicle and on the vehicle behavior with respect to others.

Yet, this micro-macro approach is more a way to analyze a mobility model than a formal description. Another way to look at mobility models is to identify two functional blocks: Motion Constraints and Traffic Generator. Motion Constraints describe how each vehicle moves (its relative degree of freedom), and is usually obtained from a topological map. Macroscopically, motion constraints are streets or buildings, but microscopically, constraints are modeled by neighboring cars, pedestrians, or by limited roads diversities either due to the type of cars or to drivers' habits. The Traffic Generator, on the other hand, generates different kinds of cars, and deals with their interactions according to the environment under study. Macroscopically, it models traffi c densities or traffi c flows, while microscopically, it deals with properties like inter-distances between cars, acceleration or braking.

The framework states that a realistic mobility model should include:

- Accurate and Realistic topological maps: Such maps should manage different densities of roads, contains multiple lanes, different categories of streets and associated velocities.

- Smooth deceleration and acceleration: Since vehicles do not abruptly break and move, deceleration and acceleration models should be considered. 
- Obstacles: We require obstacles in the large sense of the term, including both mobility and wireless communication obstacles.

- Attraction points: As any driver knows, initial and fi nal destination are anything but random. And most of the time, drivers are all driving in similar fi nal destinations, which creates bottlenecks. So macroscopically speaking, drivers move between a repulsion point towards an attraction point using a driver's preferred path.

- Simulation time: Traffi c density is not uniformly spread around the day. An heterogeneous traffi c density is always observed at some peak time of days, such as Rush hours or Special Events.

- Non-random distribution of vehicles: As it can be observed in real life, cars initial positions cannot be uniformly distributed in a simulation area, even between attraction points. Actually, depending of the Time confi guration, the density of cars at particular centers of interest, such as homes, offi ces, shopping malls are preferred.

- Intelligent Driving Patterns: Drivers interact with their environments, not only with respect to static obstacles, but also to dynamic obstacles, such as neighboring cars and pedestrians. Accordingly, the mobility model should control vehicles mutual interactions such as overtaking, traffi c jam, preferred paths, or preventive action when confronted to pedestrians.

The approach can be graphically illustrated by a concept map for vehicular mobility models, as depicted in Figure 1.

\section{Generating Mobility Models for Vehicular Networks}

Although being a promising approach, the proposed Framework in the previous section suffers from non negligeable drawbacks and limitations. Indeed, parameters defi ning the different major classes such as Topological Maps, Car Generation Engine, or Driver Behavior Engine cannot be randomly chosen, but must reflect realistic confi gurations. Therefore, due to the large complexity of such project, the research community took more simplistic directions and moved step by step.

Globally, the development of modern vehicular mobility models may be classifi ed in four different classes: Synthetic Models wrapping all models based on mathematical models, Traffic Simulators-based Models, where the vehicular mobility models are extracted from a detailed traffi c simulator, Survey-based Models extracting mobility patterns from surveys, and fi nally Trace-based Models, which generate mobility patterns from real mobility traces. A proposed classifi cation is illustrated in Fig. 2 


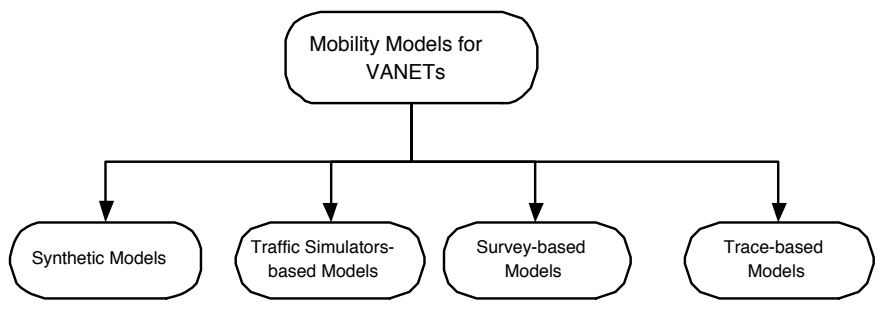

Figure 2: Classifi cation of Vehicular Mobility Models

\subsection{Synthetic Models}

The first and most well known class includes the synthetic models. Indeed, major studies have been undertaken in order to develop mathematical models reflecting a realistic physical effect. Fiore wrote a complete survey of models falling into this category. We shortly summary the basic classifi cation he developed. For a more complete version, we refer the reader to [1]. According to Fiore's classifi cation, Synthetic models may be separated in fi ve classes: stochastic models wrapping all models containing purely random motions, traffic stream models looking at vehicular mobility as hydrodynamic phenomenon, Car Following Models, where the behavior of each driver is modeled according to vehicles ahead, Queue Models which models roads as FIFO queues and cars as clients, and Behavioral Models where each movement is determined by a behavioral rules imposed by social influences for instance. Fig. 3 illustrate Fiore's classifi cation.

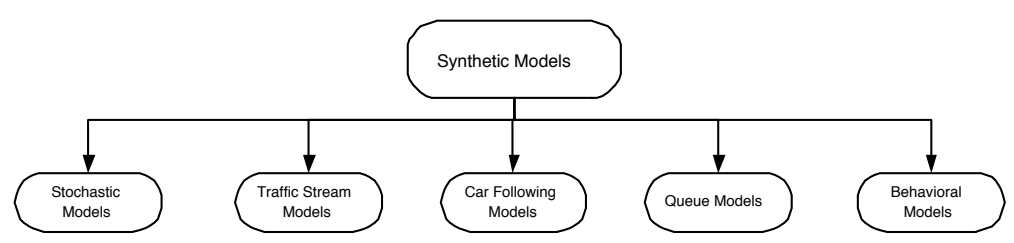

Figure 3: Classifi cation of Synthetic Mobility Models

In order to validate a mathematical model, it should be compared to real mobility. Accordingly, one solution is to gather mobility traces by large measurement campaigns then compare the patterns with those developed by the synthetic model. In [2], authors proposed to validate some key characteristics of the RWP such as average speed and rest times using real life data. The Weighted Waypoint Model (WWM) [3] is a second attempt to validate a synthetic model which has been tuned by real traces. The WWM adds the notion of preference to the random waypoint. This calibration of this preference criterion has been performed based on mobility traces obtained inside the USC campus. The HWGui [4] generates realistic time dependant highway traffi $\mathrm{c}$ patterns that have been validated against real traffi $\mathrm{c}$ in German Highways. 
A major critic from synthetic models is the lack of realism towards human behavior. Indeed, drivers are far from being machines and cannot be programmed for a specifi c behavior, but responds to stimuli and local perturbations which may have a global effect on traffi c modeling. Accordingly, realistic mobility modeling must also consider behavioral theory, social networks for instance, which makes models far from being random. Musolesi and Mascolo illustrated this approach in [5] by developing a synthetic mobility model based on social network theory, then validating it using real traces. They showed that the model was a good approximation of human movement patterns.

\subsection{Survey-based Models}

However, although the behavioral theory is able to generate macro-motion models or deviation from micro-motion models, beside the comparison with real traces, another solution is calibration by means of comparison with realistic social behavior. The major large scale available surveys come from the US department of Labor, which established surveys and gathered extensive statistics of US workers' behaviors, going from the commuting time, lunch time, traveling distance, preferred lunch politics and so forth. By including such kind of statistics into a mobility model, one is able to develop a generic mobility model able to reproduce the non random behavior observed in real daily life urban traffi c.

The UDel Mobility Model [6] falls into this category. Indeed, the mobility simulator is based on surveys from a number of research areas including time-use studies performed by the US Department of Labor and Statistics, time-use studies by the business research community, pedestrians and vehicle mobility studies by the urban planning and traffi $\mathrm{c}$ engineering communities. Based on these works, the mobility simulator simulates arrival times at work, lunch time, breaks/errands, pedestrian and vehicular dynamics (e.g., realistic speed-distance relationship and passing dynamics), and workday time-use such as meeting size, frequency, and duration. Vehicle traffi $\mathrm{c}$ is derived from vehicle traffi $\mathrm{c}$ data collected by state and local governments and models vehicle dynamics and diurnal street usage. We can also cite the Agenda-based [7] mobility model, which combines both the social activities and the geographic movements. The movement of each node is based on individual agenda, which includes all kind of activities on a specifi c day. Data from the US National Household Travel Survey has been used to obtain activity distribution, occupation distribution and dwell time distribution.

\subsection{Trace-based Models}

Another major drawback of synthetic models is that only some very complex models are able to come close to a realistic modeling of vehicular motion patterns. A different approach has therefore been undertaken. Indeed, instead of developing complex models, and then validating them using mobility traces or surveys, a crucial time could be saved by directly extracting generic mobility patterns from 
movement traces. Such approach recently became increasingly popular as mobility traces started being gathered through various measurement campaigns launched by projects such as CrawDaD [8], ETH MMTS [9], UMASSDieselNet [10], MIT Reality Mining [11], or USC MobiLib [12]. The most diffi cult part in this approach is to extrapolate patterns not observed directly by the traces. By using complex mathematical models, it is possible to predict mobility patterns not reported in the traces to some extends. Yet, the limitation is often linked to the class of the measurement campaign. For instance, if motion traces have been gathered for bus systems, an extrapolated model cannot be applied to traffi $\mathrm{c}$ of personal vehicles.

Another limitation from the creation of trace-based mobility models is the few freely available vehicular traces. The major research group are currently implementing testbeds, but the outcome might only be available in few months or years if they are even made available to the public. To corner this issue, some teams (ETH [9]), or the Los Alamos Research Labdeveloped very complex simulators, which are able to generate very realistic vehicular traces. However, due to the complexity of the simulator, the trace generation time has an order of magnitude of couples of hours or days. Then, this mobility data are usually considered as real traces for the generation or calibration of mobility models.

Tuduce and Gross in [13] present a mobility model based on real data from the campus wireless LAN at ETH in Zurich. They used a simulation area divided into squares and derive the probability of transitions between adjacent squares from the data of the access points. In [14], authors combines coarse-grained wireless traces, i.e., association data between WiFi users and access points, with an actual map of the space over which the traces were collected in order to generates a probabilistic mobility model representative of real movement. They derived a discrete time Markov Chain which not only considers the current location, but also the previous location, and also the origin and the destination of the path. However, this study does not consider correlation between nodes.

Kotz et al. [15] describe a measurement technique for extracting user mobility characteristics also from coarse-grained wireless traces. They derived the location of users over time and also emphasize popular regions. They major fi ndings was unlike standard synthetic mobility models, the speed and the pause times follow a log-normal distribution. They also confi rmed that the direction of movement closely reflect the direction of roads and walkways, and thus cannot be modeled by a uniform distribution. Similarly to [13], they ignore correlation between adjacent nodes.

In [16], user mobility are modeled by a semi-Markov process with a Markov Renewal Process associated with access point connection time instants. Unlike previous studies, this work is able to model how user mobility is correlated in time at different time scales. The authors also performed a transient analysis of the semi-markov process and extracted a timed location prediction algorithm which is able to accurately predict users future locations. This work is moreover the first attempt to characterize the correlation between movements of individual users. 
Chaintreau et al. [17] studied the inter-contact time between wireless devices carried by humans using coarse-grained wireless traces but also experimental testbeds using iMotes. Their major outbreak was that unlike the widely accepted assumption that inter-contact time follows an exponential distribution, a more realistic assumption should be that the distribution exhibit a heavy tail similar to a power law distribution. Another study ( [18]) analyzed the student contact patterns in an university campus using class time-tables and student class attendance data. A major restricted assumption has been taken, which force students to either be in classrooms or in some randomly chosen communication hubs. They showed that in this confi guration, most students experienced inter-contact time of the order of magnitude of few hours. However, unlike other studies (such as [17]), the intercontact time does not follow a power law distribution. This is where the limitation from trace-base mobility modeling appears. Indeed, this study is specifi c to class attendance, and results obtained remain also specific to the environment the study has been made.

By using traces, various research teams have therefore been able to extract mobility models that would reflect more realistically to motion we experience in real life. Moreover, a major result from trace-based mobility modeling, which is at odd with hypothesis used by synthetic models, is that the speed and pause time distributions followed a log-normal distribution, and that the inter-contact time may be modeled by a power law distribution,

\subsection{Traffi c Simulator-based Models}

By refi ning the synthetic models and going through an intense validation process using real traces or behavior surveys, some companies or research teams gave birth to realistic traffi c simulators. Developed for urban traffi c engineering, fi ne grained simulators such as PARAMICS [19], CORSIM [20], VISSIM [21] or TRANSIMS [22], are able to model urban microscopic traffi c, energy consumption or even pollution or noise level monitoring. However, those simulators cannot be used straightaway for network simulators, as no interface have been developed and traces are mutually incompatible. This, added to the commercial nature of those traffi c simulator, became the raison d'être for the development of the novel off-the-self vehicular mobility models that we are going to describe in this paper.

By developing parser between traffi c simulator traces and network simulator input fi les, the end-user gains access to validated traffi c patterns and is able to obtain a level of detail never reached by any actual vehicular mobility model. The major drawback of this approach is the confi guration complexity of those traffic $\mathrm{c}$ simulators. Indeed, the calibration usually includes tweaking a large set of parameters. Moreover, the level of detail required for vehicular network simulator may not be as demanding as that for traffi $\mathrm{c}$ analysis, as global vehicular mobility patterns and not the exact vehicular behavior are by far suffi cient in most cases. We also want to emphasize that those commercial models require the purchase of a 
license that may exceed thousands of dollars, which is a major limitation for the VANET research community.

\section{Mobility Models and Network Simulators: The Non- Speaking talking to the Deaf}

In the previous section, we described various approaches that has been undergone by the research community in order to develop realistic mobility models adapted to vehicular traffi c. Yet, in order to be used by the networking community, those models need to be made available to network simulators. And this is precisely where we fall into a kafkaien situation. The worlds of Mobility Models and Network Simulators may be compared to a non-speaking talking to deaf people. They have never been created to communicate, and even worse, they have been designed to be controlled separately, with no interaction whatsoever. When imagining the promising applications that could be obtained from vehicular networks, where networks could alter mobility, and where mobility would improve network capacity, this situation cannot be tolerated anymore if the vehicular networks community has the means of its ambitions.

Initially, mobility was seen by network simulators as random perturbations from optimum static confi gurations. Then, in order to give some control to the user on the mobility patterns, network simulators became able to load mobility scenarios. There is virtually no limitation to the complexity of the models handled by those simulators, loading scenarios extracted from traffi c simulators, or complex synthetic models for instance. However, as illustrated in Fig. 4, the models must be generated prior to the simulation and must be parsed by the simulator according to a predefi ned trace format. Then, no modifi cation of the mobility scenario is allowed.

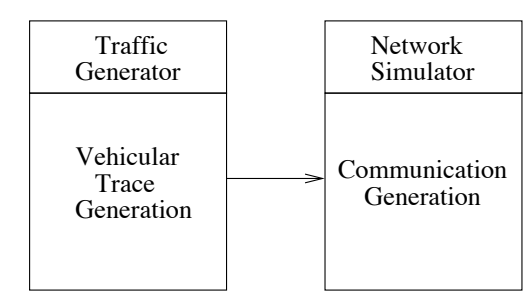

Figure 4: Interaction between Network and Traffi c Simulators: The Isolated Case

For example, VanetMobiSim [23] is able to generate realistic vehicular mobility traces in urban area as well as highway scenarios. It models car-to-car interactions and car-to-infrastructure interactions, which allows it to integrate stop signs, traffi c lights, safe inter-distance management and behavior based macro-mobility. 
It is also able to generate mobility incidents such as accidents. Moreover, it is freely available and has been validated against realistic traces obtained from CORSIM, a validated traffi c generator.

Beside the general waste of computational resources, no interaction are therefore possible between those two worlds. Unfortunately, all historical models and most of the recent realistic mobility models available to the research community fall into this category (see Section 5).

The research community then took a radically different step. If network simulators are unable to interact with mobility simulators, they should be replaced by simplistic off-the-shelf discrete even simulators which could do this task. Accordingly, new simplistic network simulator were created, where the lack of elaborated protocol stacks was compensated by a native collaboration between the networking and the mobility worlds, as depicted in Fig. 5.

MoVes [24] is an embedded system generating vehicular mobility traces and also containing a basic network simulator. The major asset of this project is its ability to partition the geographical area into clusters and parallelize and distribute the processing of the tasks from them, which improves the simulation performance. Although the mobility model reaches a suffi cient level of detail, the project's drawback is the poor network simulation, which only includes a basic physical and MAC layer architecture and totally lacks routing protocols. In [25], authors also

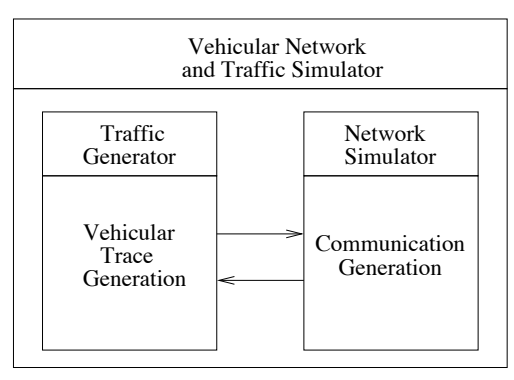

Figure 5: Interaction between Network and Traffi c Simulators: The Integrated Case

proposed an integrated vehicular and network simulator. As all solutions proposed by this approach, the authors developed their own traffi c and network simulator. The vehicular traffi $\mathrm{c}$ simulator is a synthetic model integrating basic microscopic motions where drivers may be in one of the following four modes: free driving, approaching, following, braking. A basic macroscopic model handles multi-lane and intersection management. Although being basic, this traffi c model brings suffi cient level of detail. However, the network simulator part is by far the major limitation of this project, as it is only modeled by a simplistic discrete event simulator handling a basic radio propagation and CSMA/CA MAC layer protocol.

As mentioned, the major limitation of the embedded approach is actually the poor quality of the network simulator. Indeed, this approach has been so far only 
used to study basic network effects, but could not pass the test of recent mobile ad hoc routing protocols, including realistic and standardized physical and MAC layers. And this may also be seen as a non sense, as the actual direction in network simulations is a specifi c compliance with standard protocols and computational effi ciency through parallel and distributed computing.

Another approach recently carried on is to federate existing network simulators and mobility models through a set of interfaces (see Fig. 6). For instance, MOVE [27] contains a single graphical user interface for the confi guration the mobility modeling and network simulation. However, MOVE does not itself include a network simulator, but simply parses realistic mobility traces extracted from a micro-motion model SUMO [26] into a network simulator-dependant input trace format, then generates the appropriate scripts to be loaded by the network simulator. No interaction is therefore possible between the network simulator and the mobility model.

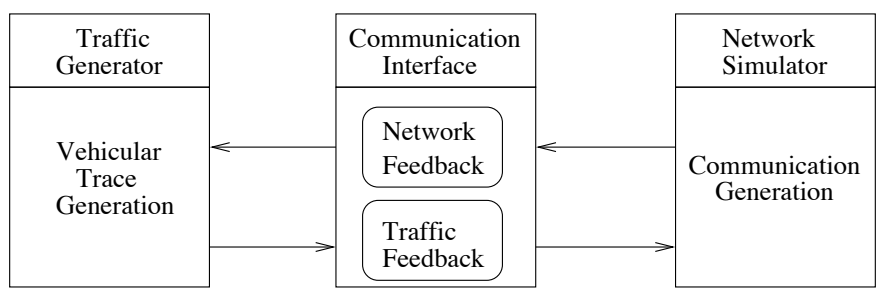

Figure 6: Interaction between Network and Traffi c Simulators: The Federated Case

A different approach, taken by Prof. Fujimoto and his group in Georgia Tech [28] is to generate a simulation infrastructure composed of two independent commercial simulation packages running in a distributed fashion over multiple networked computers. They federated a validated traffi c simulator, CORSIM, with a state-ofthe-art network simulator, QualNet, using a distributed simulation software package called the Federated Simulations Development Kit (FDK) [29] that provides services to exchange data and synchronize computations. In order to allow direct interaction between the two simulators, a common message format has been defi ned between CORSIM and QualNet for vehicle status and position information. During initialization, the transportation road network topology is transmitted to QualNet. Once the distributed simulation begins, vehicle position updates are sent to QualNet and are mapped to mobile nodes in the wireless simulation. Accordingly, unlike MOVE, both simulators work in parallel and thus may dynamically interact on each other by altering for example mobility patterns based on network flows, and vice and versa. The only limitation comes from the complex calibration of CORSIM and its large number of confi guration parameters which must be tweaked in order to fi $t$ with the modeled urban area. 
A similar solution has been taken by a team from UC Davis [30]. They developed a simulation tool federating the network simulator Swans and a synthetic traffi c model. The complex vehicular flows are based on the Nagel and Schreckenberg model, extended to include lane changing in highway scenarios. The network simulator and the traffic simulator interacts with each others by means of specific input and output messages.

Authors in [31] proposed AutoMesh, a realistic simulation framework for VANET. It is composed of a set of modules controlling all parts of a realistic simulation. It includes a Driving Simulator Module, a Radio Propagation Module, and a Network Simulator Module, all interlinked with feedback in order that any alteration made in one module influences the other modules. At the stage of the development of AutoMesh, the Driving Simulator Module only include random macro-movement and the IDM model for micro-movements. It is therefore unable to reproduce the non-uniform distribution of positions and speed usually experienced in urban area. However, the radio propagation module is very detailed, using 3D maps and digital elevation models in order to obtain a realistic radio propagation model in urban area.

Another promising approach is called TraNS [32] and also aims at federating a traffi c simulator SUMO and a network simulator ns2. Using an interface called Interpreter, traces extracted from SUMO are transmitted to ns-2, and conversely, instructions from ns-2 are sent to SUMO for traffi c tunning. TraNS will be extended to handle other network simulators such as Swans or Nab in the future. A similar project called MSIE [33] has been developped but using VISSIM instead of SUMO. This project is also more complete, as it proposes to interlink different simulators for traffi c simulation, network simulation and application simulation. The major actual limitation is the communication latency between the different simulators, and the expensive price of VISSIM. Besides, the interlinking interface itself is also not freely available at this time. Authors in [34] chose to replace VISSIM by a complete tool developed by themselves, the CARISMA traffi c simulator. Although not being as complete as VISSIM or SUMO, it allows to accurately evaluate the effects of car-to-car messaging systems in the presence of urban impediments by benefi ting from the federated approach and a "real-time" trip (re-)confi guration.

By federating independent and validated simulators, the interlinking approach is able to benefi $t$ from the best of both worlds, as state-of-the-art mobility models may be adapted to work with modern network simulators. However, it is computationally demanding, as both simulators need to be run simultaneously, and the development of the interface may not be an easy task depending on the targeted network and traffi c simulators. Nevertheless, this is probably in this direction that most of the future pioneer work will come in the fi eld of vehicular mobility modeling and networking.

The networking and mobility modeling community have a mutual interest in interacting between each others. Indeed, at the time of the promising benefi ts obtained from the various cross-layer approaches in network research, the ability to proactively or reactively act on mobility patterns in order to improve network effi - 
ciency or radio propagation, or even more promising, the ability to alter mobility patterns based on dynamically events radio transmitted will probably be a central approach in future networking research projects.

\section{A Taxonomy of existing Synthetic VANETs Mobility Mod- els}

In this Section, we provide a taxonomy of existing VANETs synthetic mobility models and simulators freely available to the research community. We fi rst introduce a set of criteria that will be able to better differentiate and classify the different synthetic models. We then provide a short summary of each model, including its assets and drawbacks, and provide its taxonomy in Table 1 and Table 2 according to the classifi cation criteria. We purposely chose not include commercial-based traffi c simulators as they cannot be freely used by the researcher working in the VANET fi eld. As a consequence, most of the federated models described in the previous section may not be included. Similarly, we can neither add Trace-based models not Survey-based Models to our taxonomy as they are extrapolated from real mobility and cannot be classifi ed according to our criteria.

\subsection{Taxonomy Criteria}

Prior to providing a classification, one need to defi ne the criteria based on which to generate the taxonomy. The proposed criteria fall in three categories: Macro-mobility, Micro-mobility, and Simulator Related.

\subsubsection{Macro-mobility Criteria}

When considering macro-mobility, we do not only take into account the road topology, but also include trip and path generation, or even the effects of points of interests, which all influence vehicles movement patterns on the road topology. We therefore defi ne the following criteria:

- Graph - The macro-motion is restricted to move on a graph.

- Initial and Destination Position - The positions may be either random, random restricted on a graph or based on a set of attraction or repulsion points.

- Trip Generation - A trip may be randomly generated between the initial and destination points, or set according to an activity sequence.

- Path Computation - Provides the algorithms used to generate the path between the points contained on the trip.

- Velocity - The simulated velocity may be uniform, smooth or road-dependant. 


\section{Graphs}

The selection of the road topology is a key factor for obtaining realistic results when simulating vehicular movements. Indeed, the length of the streets, the frequency of intersections, or the density of buildings can greatly affect important mobility metrics such as the minimum, maximum and average speed of cars, or their density over the simulated map. We categorize the graphs by the following criteria:

- User-defined - The road topology is specifi ed by listing the vertices of the graph and their interconnecting edges.

- Random - A random graph is generated, which are often Manhattan-grid, Spider, or Voronoi graphs.

- Maps - The road topology is extracted from real maps obtained from different topological standards, such as GDF, TIGER, or Arcview.

- Multi-lane - The topology includes multi-lanes, potentially allowing lane changes, or not.

We show examples of the possible topologies in Fig. 7.

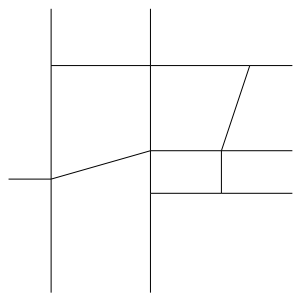

(a) User-defi ned topology

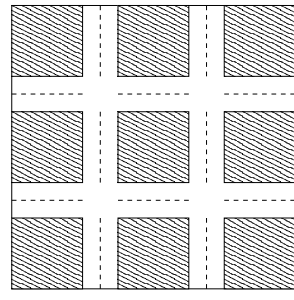

(b) Manhattan-
grid map
topology

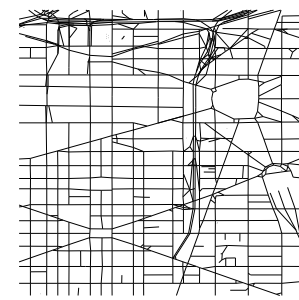

(c) TIGER map topology

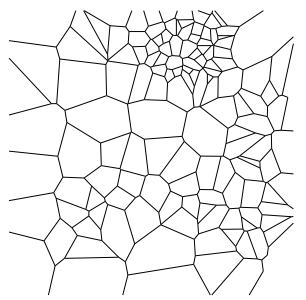

(d) Clustered Voronoi

Figure 7: Road topologies examples

\section{Attraction Points}

Attraction or Repulsion points are particular source or destination points that have a potentially attractive or repulsive feature. For instance, for a weekly morning, residential areas are repulsion points and offi ce builds are attraction points, as a large majority of vehicles are moving from the former and to the latter. We depict the use of attraction points on a user-defi ned graph in Fig. 8, where a round is for the entry/exit points of high-speed roads (thick lines), and a square for the entry/exit points of normal-speed roads (thin lines). 


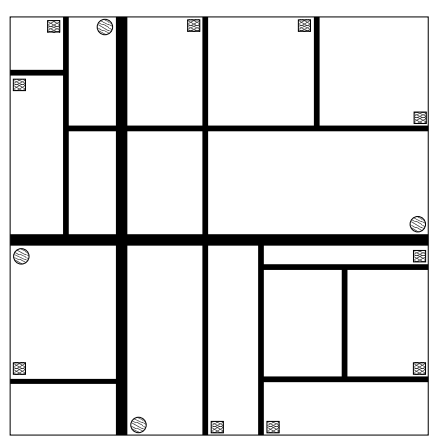

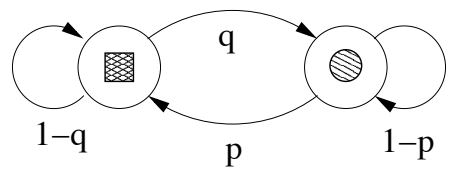

Figure 9: Activity-based Sequence between the attraction points in Fig. 8

Figure 8: Example of Attraction Points

on a User-defi ned graph

\section{Activity-based Trips}

Activity sequences generation is a further restriction in vehicles spatial and temporal distributions. A set of start and stop points are explicitly provided in the road topology description, and cars are forced to move among them. In particular, multiple sets of points of interest can be specifi ed, along with the probability matrix of a vehicle switching from one set to another. Fig. 9 illustrates an activity sequence generated from a fi rst order Markov chain between two categories of attractions points.

\subsubsection{Micro-mobility Criteria}

In the proposed taxonomy, the micro-mobility aspect uses the following criteria:

- Human Mobility Patterns - The car's internal motion and its interactions with other cars may be inspired from human motions described by mathematical models such as Car Following, or not.

- Lane Changing - Describes the kind of overtaking model implemented by the model, if any.

- Intersections - Describes the kind of intersection management implemented by the model, if any.

In this section, we shortly describe the most widely used vehicular specific micro-mobility models. We refer to [1] for a larger coverage of the different microscopic mobility models. 


\section{Car Following Models}

The car following models are a class of microscopic models that adapts a following car's mobility according to a set of rules in order to avoid contact with the lead vehicle. A general schema is illustrated in Fig. 10. Brackstone in [35] classi-

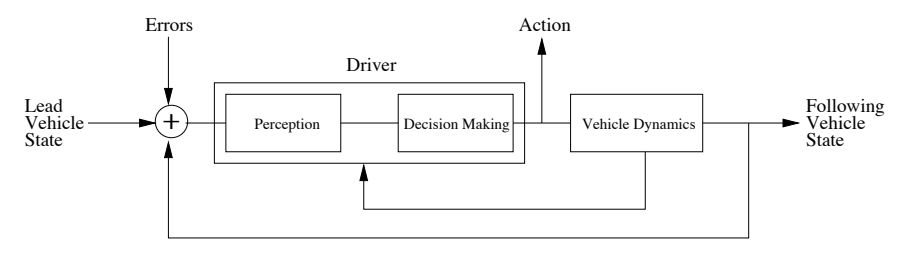

Figure 10: General Schema for Car Following Models

fi ed Car Following Models in fi ve classes: GHR Models, Psycho-Physical Models, Linear Models, Cellular Automata, Fuzzy Logic Models. A description of the differences between those models is out of scope of this paper. We refer the interested reader to [36]. We only list here the widely used models in traffi c simulations.

- Krauss Model (KM) [37]

- Nagel and Schreckenberg Model (N-SHR) [38]

- Wiedeman Psycho-Physical Model (Psycho) [39]

- General Motors Model (GM) [40]

- Gipps Model (GP) [41]

- Intelligent Driver Model (IDM) [42]

\section{Lane Changing Models}

Despite the large attention given to the driving tasks in general (such as Car Following Models), much less attention has been directed to lane changing.Modeling lane changing behavior is a more complex task. Indeed, it actually includes three parts: the need of lane changing, the possibility of lane changing, and the trajectory for lane changing. Each part is important to generate realistic lane changing models. And unlike car following models, it also needs to consider nearby cars and traffi c flow information. Most of the models are based on a Gap Acceptance threshold [43] or a set of rules [44]. But recent approaches ( $[45,46])$ also considered forced merging, behavior aspects or game theory. Lane changing is not widely considered in open vehicular mobility models. In this survey, we mostly fi nd

- Gibbs Model for Lane Changing (GP-LC) [43] and its variations

- Wiedeman Psycho-Physical Model for Lange Changing (Psycho-LC) [47] 
- MOBIL [46]

\section{Intersection Management}

Intersection management adds handling capabilities to the behavior of vehicles approaching a crossing. In most cases, two different intersection scenarios are considered: a crossroad regulated by stop signs, or a road junction ruled by traffi c lights. Nevertheless, all intersection management technics only act on the fi rst vehicle on each road, as the car following model automatically adapts the behavior of cars following the leading one. The most basic ones consider intersections as obstacles and abruptly stop, yet more complex ones, such as the IDM IM and IDM_LC [48], smoothly stop at stop-based crossing, or acquire the state of the semaphore in a traffi $\mathrm{c}$ light controlled intersection. If the color is green, passage is granted and the car maintains its current speed through the intersection. If the color is red, crossing is denied and the car is forced to decelerate and stop at the road junction. Fig. 11 illustrates the IDM_IM behavior when approaching an intersection with respect to the deceleration and the multi-lane management.

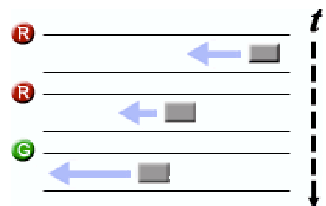

(a) Acceleration management

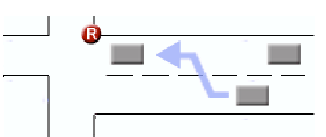

(b) Multi-lane management

Figure 11: Intersection management in IDM IM and IDM $L C$

\subsubsection{Simulator Related Criteria}

Finally, we provide these additional criteria, which are more specifi c to the mobility simulator or to the interaction with a network simulator:

- Obstacles - The model considers radio obstacles, either in the form of an obstacle topology for network simulator and a propagation computation interface for network simulators, or directly a radio propagation trace fi le.

- Visualization - The model includes a visualization tool.

- Output - Describes the kind of output generated by the mobility model, such as NS-2 or QualNet compliant traces. 
- Language - Provides the programming language on which the simulator has been developed.

\subsection{Taxonomy of Synthetic Vehicular Models}

In this section, we simultaneously provide a brief description of the major synthetic mobility models available to the vehicular networking community, and classify them in Table 1 and Table 2 according to the previously defi ned criteria. As previously mentioned, we cannot include the Trace-based nor the Survey-based models as they have been obtained from real mobility and do not fall in the taxonomy. We include some Traffic Simulator-based models if they are based on freely available traffi c simulators.

First, we point out that many realistic traffi c simulation tools, such as PARAMICS [19], CORSIM [20], VISSIM [21] or TRANSIMS [22] have been developed to analyze vehicular mobility at both microscopic and macroscopic level with a very high degree of detail. However, all the aforementioned softwares are distributed under commercial licenses, a major impediment to adoption by the academic research community. With the exception of few teams that developed parsers (e.g. [49,50]), or federated a realistic traffi c simulation tool with a network simulator (such as FDK [29]), these tools have been originally designed for traffi $\mathrm{c}$ analysis and not for generation of movement traces usable by networking simulators. Furthermore, the presence of copyrights impedes the modifi cation/extension of the sources when particular conditions, not planned by the original software, have to be simulated. For such reasons, we will not consider these tools in the following, their scope being very different from VANET mobility simulators are intended for. For a complete review and comparison of such traffi c simulation tools, the interested reader can refer to [51].

When mobility was fi rst taken into account in simulation of wireless networks, several models to generate nodes mobility patterns were proposed. The Random Waypoint model, the Random Walk model, the Reference Point Group (or Platoon) model, the Node Following model, the Gauss-Markov model, just to cite the most known ones, all involved generation of random linear speed-constant movements within the topology boundaries. Further works added pause times, reflection on boundaries, acceleration and deceleration of nodes. Simplicity of use conferred success to the Random Waypoint model in particular, however, the intrinsic nature of such mobility models may produce unrealistic movement patterns when compared to some real world behavior. Despites, random models are still widely used in the study of Mobile Ad-hoc Networks (MANETs).

As far as Vehicular Ad-hoc Networks (VANETs) are concerned, it soon became clear that using any of the aforementioned models would produce completely useless results. Consequently, the research community started seeking more realistic models. The simple Freeway model and Manhattan (or Grid) model were the initial steps, then more complex projects were started involving the generation of mobility patterns based on real road maps or monitoring of real vehicular movements in 


\begin{tabular}{|c|c|c|c|c|c|c|c|c|}
\hline & \multicolumn{8}{|c|}{ Macro-Mobility } \\
\hline & \multicolumn{4}{|c|}{ Graph } & Init/Dest & Trip & Path & Velocity \\
\hline & $\begin{array}{l}\text { User } \\
\text { Defi ned }\end{array}$ & Random & Map & Multi-lane & & & & \\
\hline $\begin{array}{l}\text { Virtual } \\
\text { Track [81] }\end{array}$ & yes & & $\begin{array}{l}\text { TIGER } \\
{[65]}\end{array}$ & no & random & $\begin{array}{l}\text { random S- } \\
\text { D }\end{array}$ & RWP & uniform \\
\hline $\begin{array}{l}\text { IMPOR- } \\
\text { TANT [61] }\end{array}$ & & Grid & & no & random & $\begin{array}{l}\text { random S- } \\
\text { D }\end{array}$ & $\begin{array}{l}\text { RWM, } \\
\text { RWalk }\end{array}$ & smooth \\
\hline $\begin{array}{l}\text { Bonn- } \\
\text { Motion } \\
{[62]}\end{array}$ & & Grid & & no & random & $\begin{array}{l}\text { random S- } \\
\text { D }\end{array}$ & RWM & uniform \\
\hline RiceM [64] & & & $\begin{array}{l}\text { TIGER } \\
\end{array}$ & no & random & $\begin{array}{l}\text { random S- } \\
\text { D }\end{array}$ & Dijkstra & uniform \\
\hline $\begin{array}{l}\text { SUMO } \\
{[26]} \\
\text { MOVE } \\
{[27]} \\
\text { TraNS [32] }\end{array}$ & yes & grid, spider & $\begin{array}{l}\text { TIGER } \\
\end{array}$ & yes & $\begin{array}{l}\text { random, } \\
\text { AP }\end{array}$ & $\begin{array}{l}\text { random S- } \\
\text { D activity }\end{array}$ & $\begin{array}{l}\text { RWalk, Di- } \\
\text { jkstra }\end{array}$ & $\begin{array}{l}\text { smooth, } \\
\text { road-dep }\end{array}$ \\
\hline $\begin{array}{l}\text { CARISMA } \\
{[34]}\end{array}$ & & & ESRI [67] & yes & random & $\begin{array}{l}\text { random S- } \\
\text { D }\end{array}$ & $\begin{array}{l}\text { Dijkstra, } \\
\text { Speed, } \\
\text { Density }\end{array}$ & $\begin{array}{l}\text { smooth, } \\
\text { road-dep }\end{array}$ \\
\hline $\begin{array}{l}\text { SHIFT } \\
\text { [69] }\end{array}$ & yes & & & yes & AP & activity & & $\begin{array}{l}\text { smooth, } \\
\text { road-dep }\end{array}$ \\
\hline $\begin{array}{l}\text { STRAW } \\
{[70]}\end{array}$ & & & $\begin{array}{l}\text { TIGER } \\
\end{array}$ & no & random & $\begin{array}{l}\text { random S- } \\
\text { D }\end{array}$ & $\begin{array}{l}\text { RWalk, Di- } \\
\text { jkstra }\end{array}$ & smooth \\
\hline $\begin{array}{l}\text { GrooveSim } \\
\text { [71] }\end{array}$ & & & $\begin{array}{l}\text { TIGER } \\
\end{array}$ & no & random & $\begin{array}{l}\text { random S- } \\
\text { D }\end{array}$ & $\begin{array}{l}\text { RWalk, Di- } \\
\text { jkstra }\end{array}$ & $\begin{array}{l}\text { uniform, } \\
\text { road-dep }\end{array}$ \\
\hline $\begin{array}{l}\text { Obstacle } \\
{[68]}\end{array}$ & & Voronoi & & no & random & $\begin{array}{l}\text { random S- } \\
\text { D }\end{array}$ & Dijkstra & uniform \\
\hline $\begin{array}{l}\text { Voronoi } \\
{[76]}\end{array}$ & & Voronoi & & no & random & $\begin{array}{l}\text { random S- } \\
\text { D }\end{array}$ & RWalk & uniform \\
\hline $\begin{array}{l}\text { GEMM } \\
{[63]}\end{array}$ & & Grid & & no & $\overline{\mathrm{AP}}$ & $\begin{array}{l}\text { random S- } \\
\text { D }\end{array}$ & RWP & uniform \\
\hline $\begin{array}{l}\text { Canu- } \\
\text { MobiSim } \\
{[72]}\end{array}$ & yes & & GDF [66] & no & $\begin{array}{l}\text { random, } \\
\text { AP }\end{array}$ & $\begin{array}{l}\text { random S- } \\
\text { D activity }\end{array}$ & $\begin{array}{l}\text { RWP, Den- } \\
\text { sity, Dijk- } \\
\text { stra }\end{array}$ & uniform \\
\hline City [74] & & Grid & & no & random & $\begin{array}{l}\text { random S- } \\
\text { D }\end{array}$ & RWM & smooth \\
\hline $\begin{array}{l}\text { Mobi- } \\
\text { REAL [77] }\end{array}$ & yes & & & no & random & $\begin{array}{l}\text { random S- } \\
\text { D }\end{array}$ & RWalk & uniform \\
\hline $\begin{array}{l}\text { SSM/TSM } \\
{[75]}\end{array}$ & & Grid & $\begin{array}{l}\text { TIGER } \\
\end{array}$ & no & random & $\begin{array}{l}\text { random S- } \\
\text { D }\end{array}$ & Dijkstra & $\begin{array}{l}\text { uniform, } \\
\text { road-dep }\end{array}$ \\
\hline $\begin{array}{l}\text { MoVES } \\
{[24]}\end{array}$ & & & $\begin{array}{l}\text { GPSTrack } \\
{[79]}\end{array}$ & no & random & & RWalk & $\begin{array}{l}\text { uniform, } \\
\text { road-dep }\end{array}$ \\
\hline $\begin{array}{l}\text { Gorgorin } \\
\text { [25] }\end{array}$ & & & TIGER & yes & random & & RWalk & smooth \\
\hline $\begin{array}{l}\text { AutoMesh } \\
{[30]}\end{array}$ & & & $\begin{array}{l}\text { TIGER } \\
\end{array}$ & yes & random & $\begin{array}{l}\text { random S- } \\
\text { D }\end{array}$ & $\begin{array}{l}\text { Dijkstra, } \\
\text { Density, } \\
\text { Speed }\end{array}$ & $\begin{array}{l}\text { uniform, } \\
\text { road-dep }\end{array}$ \\
\hline $\begin{array}{l}\text { VanetMobi- } \\
\text { Sim [23] }\end{array}$ & yes & Voronoi & $\begin{array}{l}\text { TIGER, } \\
\text { GDF }\end{array}$ & yes & $\begin{array}{l}\text { random, } \\
\text { AP }\end{array}$ & $\begin{array}{l}\text { random S- } \\
\text { D activity }\end{array}$ & $\begin{array}{l}\text { RWP, Den- } \\
\text { sity, Dijk- } \\
\text { stra, Speed }\end{array}$ & $\begin{array}{l}\text { smooth, } \\
\text { road-dep }\end{array}$ \\
\hline
\end{tabular}

Table 1: Macro-Mobility Features of the Major Vehicular Mobility Models 


\begin{tabular}{|c|c|c|c|c|c|c|c|c|}
\hline & \multicolumn{4}{|c|}{ Micro-Mobility } & \multicolumn{4}{|c|}{ Simulator Related } \\
\hline & $\begin{array}{l}\text { Human } \\
\text { Patterns }\end{array}$ & Intersection & $\begin{array}{l}\text { Lane } \\
\text { Changing }\end{array}$ & $\begin{array}{l}\text { Radio Ob- } \\
\text { stacles }\end{array}$ & $\begin{array}{l}\text { Visualization } \\
\text { Tool }\end{array}$ & Output & Platform & Remarques \\
\hline $\begin{array}{l}\text { Virtual } \\
\text { Track [81] }\end{array}$ & no & no & no & no & no & $\begin{array}{l}\text { ns-2, glo- } \\
\text { moSim, } \\
\text { QualNet }\end{array}$ & $\mathrm{C}++$ & \\
\hline $\begin{array}{l}\text { IMPOR- } \\
\text { TANT [61] }\end{array}$ & CFM & no & no & no & no & ns-2 & $\mathrm{C}++$ & $\begin{array}{l}\text { unrealistic } \\
\text { CFM }\end{array}$ \\
\hline $\begin{array}{l}\text { Bonn- } \\
\text { Motion } \\
{[62]}\end{array}$ & no & no & no & no & yes & $\begin{array}{l}\text { ns-2, glo- } \\
\text { moSim, } \\
\text { QualNet }\end{array}$ & Java & \\
\hline RiceM [64] & no & no & no & no & no & $\begin{array}{l}\text { ns-2, glo- } \\
\text { moSim, } \\
\text { QualNet }\end{array}$ & $\mathrm{C}++$ & \\
\hline $\begin{array}{l}\text { SUMO } \\
{[26]} \\
\text { MOVE } \\
{[27]} \\
\text { TraNS [32] }\end{array}$ & $\begin{array}{l}\text { CFM } \\
\text { (Krauss) }\end{array}$ & stoch turns & no & no & yes & $\begin{array}{l}\text { ns-2, glo- } \\
\text { moSim, } \\
\text { QualNet }\end{array}$ & $\mathrm{C}++$ & $\begin{array}{l}\text { federated } \\
\text { traf/net } \\
\text { simulator, } \\
\text { validated } \\
\text { micro- } \\
\text { model }\end{array}$ \\
\hline $\begin{array}{l}\text { CARISMA } \\
{[34]}\end{array}$ & $\begin{array}{l}\text { CFM } \\
\text { (Krauss) }\end{array}$ & stop signs & no & yes & yes & $\begin{array}{l}\text { ns-2, glo- } \\
\text { moSim, } \\
\text { QualNet }\end{array}$ & $\mathrm{C}++$ & $\begin{array}{l}\text { federated } \\
\text { traf/net } \\
\text { simulator }\end{array}$ \\
\hline $\begin{array}{l}\text { SHIFT } \\
\text { [69] }\end{array}$ & CFM & no & $\mathrm{LC}$ & no & yes & none & $\mathrm{C}++/ \mathrm{SHIFT}$ & $\begin{array}{l}\text { confi gurable } \\
\text { CFM/LC }\end{array}$ \\
\hline $\begin{array}{l}\text { STRAW } \\
\text { [70] }\end{array}$ & $\begin{array}{l}\text { CFM } \\
\text { (Nagel } \\
\text { Schreck) }\end{array}$ & $\begin{array}{l}\text { traffic } \\
\text { lights, } \\
\text { signs }\end{array}$ & no & no & no & Swans & $\begin{array}{l}\text { JiST- } \\
\text { Swans }\end{array}$ & \\
\hline $\begin{array}{l}\text { GrooveSim } \\
\text { [71] }\end{array}$ & no & no & no & & yes & none & $\mathrm{C}++$ & \\
\hline $\begin{array}{l}\text { Obstacle } \\
{[68]}\end{array}$ & no & no & no & yes & yes & $\begin{array}{l}\text { ns-2, glo- } \\
\text { moSim, } \\
\text { QualNet }\end{array}$ & $\mathrm{C}++$ & \\
\hline $\begin{array}{l}\text { VoronoiM } \\
{[76]}\end{array}$ & no & no & no & no & no & ns-2 & $\mathrm{C}++$ & \\
\hline $\begin{array}{l}\text { GEMM } \\
{[63]}\end{array}$ & no & no & no & no & no & ns-2 & Java & \\
\hline $\begin{array}{l}\text { Canu- } \\
\text { MobiSim } \\
{[72]}\end{array}$ & IDM & no & no & yes & yes & $\begin{array}{l}\text { ns-2, glo- } \\
\text { moSim, } \\
\text { QualNet, }\end{array}$ & Java & \\
\hline City [74] & IDM & stoch turns & no & no & yes & ns-2 & $\mathrm{C}++$ & \\
\hline $\begin{array}{l}\text { MobiREAL } \\
\text { [77] }\end{array}$ & $\mathrm{CPE}$ & no & no & yes & yes & GTNetS & $\mathrm{C}++$ & $\begin{array}{l}\text { pedestrian } \\
\text { mobility }\end{array}$ \\
\hline $\begin{array}{l}\text { SSM/TSM } \\
{[75]}\end{array}$ & no & $\begin{array}{l}\text { traffi c } \\
\text { lights, } \\
\text { traffi c } \\
\text { signs } \\
\end{array}$ & no & no & no & $\begin{array}{ll}\mathrm{ns}-2 \\
\mathrm{n}\end{array}$ & $\mathrm{C}++$ & \\
\hline $\begin{array}{l}\text { MoVES } \\
{[24]}\end{array}$ & $\begin{array}{l}\text { CFM (Psy- } \\
\text { cho) }\end{array}$ & $\begin{array}{l}\text { random } \\
\text { traffi c } \\
\text { lights, } \\
\text { traffi c } \\
\text { signs }\end{array}$ & no & no & yes & none & $\mathrm{C}++$ & $\begin{array}{l}\text { integrated } \\
\text { traf/net } \\
\text { simulator }\end{array}$ \\
\hline $\begin{array}{l}\text { Gorgorin } \\
{[25]}\end{array}$ & $\begin{array}{l}\text { CFM (Psy- } \\
\text { cho) }\end{array}$ & $\begin{array}{l}\text { traffic } \\
\text { lights, } \\
\text { traffic } \\
\text { signs }\end{array}$ & $\begin{array}{l}\text { CFM } \\
\text { (Psycho- } \\
\text { LC) }\end{array}$ & no & yes & none & $\mathrm{C}++$ & $\begin{array}{l}\text { integrated } \\
\text { traf/net } \\
\text { simulator, } \\
\text { validated } \\
\text { micro- } \\
\text { model }\end{array}$ \\
\hline $\begin{array}{l}\text { AutoMesh } \\
{[30]}\end{array}$ & IDM & stop signs & no & yes & yes & $\begin{array}{l}\text { ns-2, glo- } \\
\text { moSim, } \\
\text { QualNet }\end{array}$ & $\mathrm{C}++$ & $\begin{array}{l}\text { federated } \\
\text { traf/net } \\
\text { simulator }\end{array}$ \\
\hline $\begin{array}{l}\text { VanetMobi- } \\
\text { Sim [23] }\end{array}$ & $\begin{array}{l}\text { IDM, } \\
\text { IDM_IM, } \\
\text { IDM_LC }\end{array}$ & $\begin{array}{l}\text { traffic } \\
\text { signs, } \\
\text { traffic } \\
\text { lights }\end{array}$ & MOBIL & yes & yes & $\begin{array}{l}\text { ns-2, } \\
\text { qualNet, } \\
\text { glomoSim }\end{array}$ & Java & $\begin{array}{l}\text { validated } \\
\text { macro/micro } \\
\text { model }\end{array}$ \\
\hline
\end{tabular}

Table 2: Micro-Mobility Features of the Major Vehicular Mobility Models 
cities. However, in most of these models, only the macro-mobility of nodes was considered. Although car-to-car interactions are a fundamental factor to take into account when dealing with vehicular mobility [52], little or no attention was paid to micro-mobility. More complete and detailed surveys of mobility models can be found in literature [53-56].

Recently, new open-source tools became available for the generation of vehicular mobility patterns. Most of them are capable of producing traces for network simulators such as $n s-2$ [57], GloMoSim [58], QualNet [59], or OpNet [60]. In the rest of this section, we review some of these tools, in order to understand their strengths and weaknesses.

The IMPORTANT tool [61], and the BonnMotion tool [62] implement most of the random mobility models presented in [53], including the Manhattan model. This model restrict nodes macro-mobility on a grid, while the micro-mobility contains a Car Following Model. The BonnMotion does not consider any micromobility. When related to our proposed framework, we can easily see that the structure of both tools is defi nitely too simple to represent realistic motions, as they only model basic motion constraints and hardly no micro-mobility.

The GEMM tool [63] is an extension to BonnMotion's and improves its traffi c generator by introducing the concepts of human mobility dynamics, such as Attraction Points (AP), Activity, or Roles. Attraction points reflect a destination interest to multiple people, such as grocery stores or restaurants. Activities are the process of moving to an attraction point and staying there, while roles characterize the mobility tendencies intrinsic to different classes of people. While the basic concept is interesting, its implementation in the tool is limited to a simple enhanced RWM between APs. It however represents an initial attempt to improve the realism of mobility models by considering human mobility dynamics.

The MONARCH project [64] proposed a tool to extract road topologies from real road maps obtained from the TIGER [65] database. The possibility of generating topologies from real maps is considered in the framework, however the complete lack of micro-mobility support makes it diffi cult to represent a complete mobility generator. Indeed, this mobility model is simply a Random Waypoint Model restricted on a graph extracted from real topological cities. Although it brings some spatial correlations, it absolutely lacks time, car-to-car and car-toinfrastructures correlations. Besides, the authors showed that their model was having similar patterns than the RWM.

The Obstacle Mobility Model [68] takes a different approach in the objective of obtaining a realistic urban network in presence of building constellations. Instead of extracting data from TIGER fi les, the simulator uses random building corners and voronoi tessellations in order to defi ne movement paths between buildings. It also includes a radio propagation model based on the constellation of obstacles. According to this model, movements are restricted to paths defi ned by the Voronoi graph.

The Simulation of Urban MObility (SUMO) [26] is an open source, highly portable, microscopic road traffi c simulation package designed to handle large road 
networks. The car microscopic movement model in SUMO is a car following model and includes a stochastic traffi $\mathrm{c}$ assignment modeled by a probabilistic route choice according to driver models. SUMO contains parsers for various topologies, ranging from TIGER, Arcview, or even to VISSIM. Routes assignment may also be imported from various sources. However, at that time, SUMO is not able to output traces straightforwardly usable by network simulators.

The Mobility Model Generator for Vehicular Networks (MOVE) was recently presented [27]. It is a simple parser for the SUMO and enhances SUMO's complex confi guration with a nice and effi cient GUI. MOVE also contains a parser to generate traces usable by network simulators such as ns-2 or QualNet.

SUMO is also the root functionality of TraNS [32], a federated model including ns2. Using an interface called Interpreter, traces extracted from SUMO are transmitted to ns-2, and conversely, instructions from ns-2 are sent to SUMO for traffi $\mathrm{c}$ tunning. Accordingly, interactions between the vehicular traffi $\mathrm{c}$ and network may be implemented.

Another important microscopic mobility simulator is the SHIFT Traffi c Simulator [69]. It has been developed by the PATH Project at the UC Berkley, and is now a well established micro-mobility simulator that generates the trajectories of vehicles driving according to validated models on realistic road networks. More specifi cally, SHIFT is a new programming language with simulation semantics and was used in SmartAHS as means of specifi cation, simulation and evaluation framework for modeling, control and evaluation of Automated Highway Systems (AHS). The major limitation of this simulator is its limitation to the modeling of segments of highways and its lack of complete topology modeling.

The CARISMA traffi c simulator [34] is a realistic simulator containing microscopic and macroscopic features. It implements the Krauss's car following model, adds a stop sign intersection management, imports real topological maps in ESRI standard [67]. It furthermore provides a real-time trip management, which is a very interesting feature for the evaluation of car-to-car messaging. This model has also been interlinked with ns-2 for realistic evaluations of vehicle-to-vehicle messaging systems. One major limitation comes from the ESRI shape files, which are not publicly available unless you buy some ESRI products. Moreover, lane changing models and complex intersection managements are not considered at that time.

The Street Random Waypoint (STRAW) tool [70] is a mobility simulator based on the freely available Scalable Wireless Ad Hoc Network Simulator (SWANS). Under the point of view of vehicular mobility, it provides urban topologies extractions from the TIGER database, as well as micro-mobility support. STRAW is also one of the few mobility tools to implement a complex intersection management using traffi c lights and traffi c signs. Thanks to this, vehicles are showing a more realistic behavior when reaching intersection. The concept behind STRAW is very similar to the framework described in section 2, as it contains accurate mobility constraints as well as a realistic traffic generator engine. STRAW also includes several implementations of transport, routing and media access protocols, since 
they are not present in the original SWANS software. The main drawback of the tool is the very limited diffusion of the SWANS platform.

The GrooveSim tool [71] is a mobility and communication simulator, which again uses fi les from the TIGER database to generate realistic topologies. Being a self-contained software, GrooveSim neither models vehicles micro-mobility, nor produces traces usable by network simulators. The interesting feature of this model is the non uniform distribution of vehicles speeds. Indeed, motion constraints such as speed limitations, often force vehicles to give up in their effort to reach the velocity initially set by the model. Although that is might look as a straightforward pattern, this type of motion constraints is, at this time, considered only by few simulators. GrooveSim includes four types of velocity models, where the most interesting is the road-based velocity when used in conjunction with a shortest trip path generation. The authors illustrated how vehicles were naturally choosing the roads with the highest speed limitations while on their journey. The main drawback of this tool is however its lack of a micro-mobility model as well as the lack of mobility traces for network simulators.

The CanuMobiSim tool [72] is a tool for the generation of movement traces in a variety of conditions. Extrapolation of real topologies from detailed Geographical Data Files (GDF) are possible, many different mobility models are implemented, a GUI is provided, and the tool can generate mobility traces for $n s-2$ and GloMoSim. Unlike many other tools, the CanuMobiSim tool keeps micro-mobility in consideration, implementing several car-to-car interaction models such as the Fluid Traffic Model, which adjusts the speed given vehicles local density, or the Intelligent Driver Model (IDM), which adapts the velocity depending on movements between neighboring vehicles. Also unlike other tools, CanuMobiSim includes a complex traffi $\mathrm{c}$ generator that can either implements basic source-destination paths using Dijkstra-like shortest path algorithms, or similarly to the GEMM, it can model trips between Attraction Points depending on the class of users' specifi c motion patterns. This solution is actually the only fully implemented and available solution considering heterogeneous classes of user and destinations. In order to improve its modeling capability, CanuMobiSim has even been recently extended (see [73]) by the same authors and now includes radio propagation information for ns-2 and GloMoSim/QualNet.

In recent months, a couple of research teams proposed a new set of simulators that comes closer to the objective to accurately model vehicles' specifi c motions. The fi rst one is the City Model [74]. It has been basically designed for routing protocols testing and no network simulator traces are provided. This model includes a basic micro-mobility model composed of the IDM and a simplistic crossing management. Crossings are modeled like obstacles, where a car needs to reduce its speed and stop before the crossing. Then, the vehicle changes its direction according to a particular probability. This simulator mostly lacks modularity mostly due to its unique grid-based macro-mobility constraints, to the restriction to stochastic turns, and to the lack macro-mobility patterns based on human mobility dynamics.

The second is the SSM/TSM model [75]. It represents actually two different 
mobility models, a Stop Sign Model and a Traffic Sign Model. The motion constraints part is dealt using a TIGER parser, while the traffi c generator includes the Car Following Model. As GrooveSim, both SSM and TSM include a roaddependent velocity distribution. However, this model goes farer than GrooveSim, since it contains a basic traffic generator which makes its mobility traces more realistic than GrooveSim's. And similarly to STRAW, SSM/TSM has been specifically designed to model vehicles' motions at intersections. The authors managed to show how a basic intersection management such as a simple stop sign was able to produce a clustering effect at those intersection. In urban environment, this effect is better known under the name Traffic Jam, and is hardly represented in most of the actual simulators. But similarly to the City Model, the SSM/TSM also lacks macro-mobility patterns based on human mobility dynamics.

The Voronoi Model [76] is an illustration of how voronoi graphs proposed by some simulators could be refi ned and improved to generate smoother roads. Unlike other mobility models including voronoi tessellations, this Voronoi Model does not model roads as graph edges, but as voronoi channels. A voronoi channel is a spatial area obtained after multiple application of a Voronoi Tessellation algorithm. It provides a global moving direction, while keeping some degree of liberty in the local direction patterns. Most of this model contributions are on the improvement of the motion constraints component as a promising random topology generator, while the traffi c generator engine is a simple implementation of a Random Walk within each voronoi channel. However, this model's absolute lack of micro-mobility considerations and macro-mobility patterns based on human mobility dynamics, makes it unrealistic for vehicular mobility modeling.

All models presented in this section so far claims to be able to model realistic vehicular motion patterns. However, with the exception of SHIFT, none of them formally validated their patterns agaist real vehicular traces, or validated traffic $\mathrm{c}$ simulators. VanetMobiSim [23], on the other hand, is the only synthetic model so far, which motion patterns for urban and highways environments have been validated. Indeed, the authors compared the traces obtained from VanetMobiSim and from CORSIM on similar urban confi gurations. They managed to show that the spatial distributions, the speed distributions, and the traffi c shock waves generated by both models were similar. As CORSIM has been formally validated against real urban traces, so are VanetMobiSim's.

VanetMobiSim models car-to-car and car-to-infrastructure interactions, allowing it to integrate stop signs, traffi c lights, safe inter-distance management and behavior based macro-mobility including human mobility dynamics. It also includes various road topology defi nition, ranging from realistic GDF [66] or TIGER [65], to user-defi ned or random topologies. It lets the user defi ne the trip generation between random source-destination, to activity-based trips. Moreover, the path used on the defi ned trip is also confi gurable between a Dijkstra shortest-path, a roadspeed shortest path and a density-based shortest path. It fi nally generates traces for various network simulators, as well as a special Universal Trace Format, which is 
simply composed of

Universal Trace Format: time node_id pos_X pos_Y velocity acceleration

which may be used by any tool capable of parsing that kind of trace fi le. VanetMobiSim is at that time one of the most realistic and confi gurable synthetic model for the generation of vehicular motion patterns.

A special attention should also be brough to a novel solution named MobiREAL [77]. Although that it focuses on the modeling of pedestrian mobility, its strict compliance with the proposed framework and its novel approach of cognitive modeling makes it very promising for a future extension to vehicular mobility. The most interesting features is that MobiREAL enables to change a node or a class of nodes' mobility behavior depending on a given application context. At this time, only CanuMobiSim, VanetMobisim and MobiREAL are able to include this feature. This particular application context is modeled by a Condition Probability Event $(C P E)$, a probabilistic rule-based mobility model describing the behavior of mobile nodes, which is often used in cognitive modeling of human behavior. As most of recent mobility models, MobiREAL is able to include geographical informations. Moreover, it is also able to use this information to generate obstacles and more specifi cally it is able to model radio's interference and attenuations on the simulation fi eld. With CanuMobiSim's extension and the Obstacle model, they are the only models that are able to both generate motion traces and signal attenuation information. MobiREAL's major drawback at this time is the limited diffusion of Georgia Tech Network Simulator (GTNets) and the manual confi guration of all necessary parameters, which requires a full recompilation of the simulator at each reconfi guration.

Recently, new approaches appears in realistic scalable simulations of vehicular mobility. In [24], the authors created MoVes, a complex mobility generator on top of Artis [78], a scalable distributed simulation middleware. MoVes features cars following models, drivers' characterization, intersection management and includes a parser module to include GPS maps using the GPS TrackMaker program [79]. However, unlike our project, MoVes does not include any lane changing model, and no realistic path generation is supported.

Gorgorin [25] also integrated a network and a mobility simulator. Although the idea looks promising, the major flow at this time is the relative simplicity of both simulators. Indeed, although the mobility model is able to import TIGER maps and includes a similar micro mobility model to VISSIM, it does not consider any macro-mobility aspect. Moreover, similarly to MoVes, the network simulator also suffers from its simplistic architecture and from its poor diffusion compared to QualNet, OpNet or Ns-2.

The UDel Models [6] are a set of mobility and radio propagation models generated for detailed large-scale urban mesh networks. The urban mobility part is signifi cantly different from all the previous approaches, as detailed urban vehicular and pedestrian mobility is based on surveys. Indeed, urban planning and the 
US Department of Labor generated a large database of statistics on time use or human mobility preferences. The generated simulator also considers a detailed urban propagation model and includes an accurate map builder capable of parsing GIS dataset and adding realistic radio obstacles.

\section{Conclusion}

As a prospective technology, Vehicular Ad Hoc Networks (VANETs) have recently been attracting an increasing attention from both research and industry communities. One of the fastest growing fi eld of interest in VANETs is safety, where communications are exchanged in order to improve the driver's responsiveness and safety in case of road incidents. VANETs characteristics are a higher mobility and a limited degree of freedom in the mobility patterns. Such particular features make standard networking protocols ineffi cient or unusable in VANETs. Accordingly, one of the critical aspect when testing VANETs protocols is the use of mobility models that reflect as closely as possible the real behavior of vehicular traffi c. In this paper, we fi rst presented a framework which should be followed for the generation of realistic vehicular mobility patterns, then we disserted on the different approaches in vehicular mobility modeling and proposed a classifi cation of vehicular mobility models according to the technics used for their generation. We fi nally described the most popular models available to the research community at this time, and provided their detailed taxonomy according to criteria based on natural building blocks required for realistic vehicular mobility modeling.

Unlike MANETs, the major objective of VANET protocols is a direct alteration of the traffi c patterns for safety or trip optimization. Accordingly, we also described the new trend to interlink traffi $\mathrm{c}$ and network simulators in order to create a crosslayer collaboration between routing and mobility schemes. As far as the authors are concerned, this is the fi rst article which clearly addresses this issue in perspective to other approaches, and provides an insight of the future research directions in joint traffi $\mathrm{c}$ and network simulations.

The aim of this survey is to facilitate the comprehensive understanding of the emerging development of realistic vehicular traffi c generators, the different methods, their justifi cations, and the interlinking with network simulators. This could be a good guideline for people interested in understanding the unique relationship between traffi c models and network protocols in vehicular networks. This article also provided a large coverage of the most popular mobility models for vehicular networks, and could thus be a good starting point for people starting in this fi eld or desiring to increase their knowledge in Vehicular Ad Hoc Mobility Modeling. 



\section{References}

[1] Marco Fiore, "Mobility Models in Inter-Vehicle Communications Literature", Technical Report, November 2006.

[2] Andres Rojas, Philip Branch, Grenville Armitage, "Experimental validation of the random waypoint mobility model through a real world mobility trace for large geographical areas", in Proc. of the 8th ACM international symposium on Modeling, analysis and simulation of wireless and mobile systems, Montreal, Canada, October 2005.

[3] W. Hsu, K. Merchant, H. Shu, C. Hsu, A. Helmy, "Weighted Waypoint Mobility Model and its Impact on Ad Hoc Networks", in Proc. of th ACM Mobile Computer Communications Review (MC2R), Jan 2005.

[4] The HWGui Project - Visualizing, Evaluating and Transforming Movement Patterns of Vehicles on Highways, http://www. informatik. uni-mannheim.de/pi4.data/content/projects/hwgui

[5] Mirco Musolesi and Cecilia Mascolo, "A Community Based Mobility Model for Ad Hoc Network Research" In Proc. of the 2nd ACM/SIGMOBILE International Workshop on Multi-hop Ad Hoc Networks: from theory to reality (REALMAN'06), Florence, Italy, May 2006.

[6] UDel Models For Simulation of Urban Mobile Wireless Networks, http: //udelmodels.eecis.udel.edu/

[7] Qunwei Zheng Xiaoyan Hong Jun Liu, "An Agenda-based Mobility Model" in Proc. of the 39th IEEE Annual Simulation Symposium (ANSS-39-2006), Huntsville AL, April 2006.

[8] Community Resource for Archiving Wireless Data At Dartmouth (Crawdad), http://crawdad.cs.dartmouth.edu

[9] Realistic Vehicular Traces http://1st.inf.ethz.ch/ad-hoc/car-traces

[10] UMass Diverse Outdoor Mobile Environment (DOME), http://prisms.cs.umass.edu/dome

[11] MIT Media Lab: Reality Mining, http://reality.media.mit.edu

[12] Community-wide Library of Mobility and Wireless Networks Measurements, http://nile.usc.edu/MobiLib

[13] Cristian Tuduce and Thomas Gross, "A Mobility Model Based on WLAN Traces and its Validation", in Proc. of the IEEE INFOCOM 2005, Miami, March 2005. 
[14] Jungkeun Yoon, Brian D. Noble, Mingyan Liu, Minkyong Kim, "Building realistic mobility models from coarse-grained traces", in Proc. of the ACM International Conference On Mobile Systems, Applications And Services (MobiSys'06), pp. 177-190, 2006.

[15] Minkyong Kim, David Kotz and Songkuk Kim, "Extracting a Mobility Model from Real User Traces", in Proc. of the 26th Annual IEEE Conference on Computer Communications (INFOCOM'06), Barcelona, April 2006.

[16] J. Hou et al., "Modeling Steady-state and Transient Behaviors of User Mobility: Formulation, Analysis, and Application", in Proc. of the the 7th ACM International Symposium on Mobile Ad Hoc Networking and Computing (MOBIHOC'06), Florence, May 2006.

[17] Augustin Chaintreau et al., "Impact of Human Mobility on the Design of Opportunistic Forwarding Algorithms" in Proc. of the 26th Annual IEEE Conference on Computer Communications (INFOCOM'06), Barcelona, April 2006.

[18] V. Srinivasan et al., "Analysis and Implications of Student Contact Patterns Derived from Campus Schedules", in Proc. of the The 12th ACM International Conference on Mobile Computing and Networking (MOBICOM'06), Los Angeles, 2006.

[19] Paramics: Microscopic Traffic Simulation, http://www paramics-online.com/

[20] CORSIM: Microscopic Traffic Simulation Model, http:// www-mctrans.ce.ufl.edu/featured/TSIS/Version5/ corsim.htm

[21] ptv simulation - VISSIM, http://www.english.ptv.de/cgi-bin/ traffic/traf_vissim.pl

[22] TRANSIMS, transims.tsasa.lanl.gov

[23] VanetMobiSim Project Home Page, http: //vanet.eurecom. fr.

[24] L. Bononi, M. Di Felice, M. Bertini, E. Croci, "Parallel and Distributed Simulation of Wireless Vehicular Ad Hoc Networks", in proc. of the ACM/IEEE International Symposium on Modeling, Analysis and Simulation of Wireless and Mobile Systems (MSWiM), Torresmolinos, Spain, 2006.

[25] C. Gorgorin, V. Gradinescu, R. Diaconescu, V. Cristea, L. Ifode, "An Integrated Vehicular and Network Simulator for Vehicular Ad-Hoc Networks", in Proc. of the European Simulation and Modelling Conference (ESM), Bonn, Germany, May 2006.

[26] SUMO - Simulation of Urban MObility, http://sumo.sourceforge.net 
[27] F. Karnadi, Z. Mo, K.-C. Lan, "Rapid Generation of Realistic Mobility Models for VANET", in Proc. of the IEEE Wireless Communication and Networking Conference (WCNC'07), March 2007.

[28] H. Wu, J. Lee, M. Hunter, R. M. Fujimoto, R. L. Guensler, and J. Ko, "Simulated Vehicle-to-Vehicle Message Propagation Effi ciency on Atlanta's I-75 Corridor," in Transportation Research Board Conference Proceedings, Washington D.C., 2005.

[29] The Federated Simulations Development Kit (FDK), http: //www-static.cc.gatech.edu/computing/pads/fdk.html

[30] Andrew Chen, et al., "Impact of Transmission Power on TCP Performance in Vehicular Ad Hoc Networks" in Proc. of the 4th IEEE/IFIP Wireless On demand Networks and Services, pp. 65-71, Austria 2007.

[31] Rama Vuyyuru, Kentaro Oguchi, "Vehicle-to-Vehicle Ad Hoc Communication Protocol Evaluation using Simulation Framework", in Proc. of the 4th IEEE/IFIP Wireless On demand Networks and Services, pp. 100-106, Austria 2007.

[32] Traffi c and Network Simulation Environment http://wiki.epfl.ch/trans/

[33] Multiple Simulator Interlinking Environment for $\mathrm{C} 2 \mathrm{CC}$ in VANETs, http://www.cn.uni-duesseldorf.de/projects/MSIE

[34] C. Schroth et al., "Simulating the traffi c effects of vehicle-to-vehicle messaging systems", in Proc. of the 5th International Conference on ITS Telecommunications, Brest, France, 2005.

[35] M. Brackstone, M. McDonald, "Car-following: a historical review", Transportation Research Part F: Traffi c Psychology and Behaviour, Volume 2, Number 4, pp. 181-196, 1999.

[36] Sakda Panwai, Hussein Dia, "Comparative Evaluation of Microscopic Car Following Behavior", in IEEE Transactions on Intelligent Transportation Systems, Vol. 6 No. 3, pp. 314-325, September 2005.

[37] Stefan Krauss, Peter Wagner, Christian Gawron, "Metastable States in a Microscopic Model of Traffi c Flow" Physical Review E, volume 55, number 304, pages 55-97; May, 1997.

[38] Kai Nagel and Michael Schreckenberg, "A cellular automaton model for freeway traffi c" Journal de Physic I, vol. 1992, no. 2, pp. 2221-2229, 1992.

[39] R. Wiedemann, "Simulation des Straenverkehrsflusses", in Schriftenreihe Heft 8, Institute for Transportation Science, University of Karlsruhe, Germany, 1974. 
"Traffi c Dynamics: Studies in Car Following", Operations Research, Vol. 6, No. 2, pp. 165-184, 1958.

[40] Robert E. Chandler, Robert Herman, Elliott W. Montroll, "Traffi c Dynamics: Studies in Car Following”, Operations Research, Vol. 6, No. 2, pp. 165-184, 1958.

[41] P. G. Gipps, "A behavioural car following model for computer simulation", Transportation Research Board 15, pp. 105?111, 1981.

[42] M. Trieber, A. Hennecke, D. Helbing, "Congested traffi c states in empirical observations and microscopic simulations", Phys. Rev. E 62, Issue 2, August 2000 .

[43] P. G. Gipps, "A Model for the Structure of Lane Changing Decisions", Transportation Research Board 20, pp. 107?120. 1986.

[44] D. Chowdhury, D.E. Wolf, and M. Schreckenberg, "Particle hopping models for two-lane traffi c with two kinds of vehicles:Effects of lane-changing rules", Physica A, pp. 235-687, 1997.

[45] K, I, Ahmed, "Modeling Drivers' Acceleration and Lane Changing Behavior", Ph.D Thesis, MIT Press, USA, 1999.

[46] M. Treiber, D. Helbing, "Realistische Mikrosimulation von Strassenverkehr mit einem einfachen Modell", 16th Symposium Simulationstechnik ASIM 2002, Rostock, September 2002.

[47] R. Wiedemann, "Modeling of RTI-Elements on multi-lane roads", in $A d$ vanced Telematics in Road Transport, edited by the Commission of the European Community, vol. DG XIII, 1991.

[48] M. Fiore, J. Harri, F. Filali, and C. Bonnet, "Vehicular mobility simulation for VANETs" in Proc of the 40th IEEE/SCS Annual Simulation Symposium (ANSS'07), March 25 - March 29, 2007, Norfolk, USA.

[49] Jijun Yin, Tamer ElBatt, Gavin Yeung, Bo Ryu, Stephen Habermas, "Performance Evaluation of Safety Applications over DSRC Vehicular Ad Hoc Networks", in Proc. of the 1st ACM International Workshop on Vehicular Ad Hoc Networks (VANET), October 2004.

[50] Hao Wu, Richard Fujimoto, Randall Guensler, Michael Hunter, "MDDV: Mobility-Centric Data Dissemination Algorithm for Vehicular Networks", in Proc. of the 1st ACM International Workshop on Vehicular Ad Hoc Networks (VANET), October 2004.

[51] S. Boxill and L. Yu, "An Evaluation of Traffi c Simulation Models for Supporting ITS Development", Technical Report 167602-1, Texas Southern University, October 2000. 
[52] D. Helving, "Traffi c and Related Self-driven Many-particles Systems", Rev. Modern Physics, Vol. 73, pp. 1067-1141, 2001.

[53] T. Camp, J. Boleng, V. Davies, "A Survey of Mobility Models for Ad Noc Network Research", Wireless Communications and Mobile Computing, Vol. 2, Issue 5, pp. 483-502, September 2002.

[54] F. Bai, A. Helmy, "A Survey of Mobility Modeling and Analysis in Wireless Ad Hoc Networks", Book chapter in Wireless Ad Hoc and Sensor Networks, Kluwer academic Publishers, June 2004.

[55] J.-Y. Le Boudec, M. Vojnovic, "Perfect Simulation and Stationarity of a Class of Mobility Models", IEEE INFOCOM 2005, Miami, FL, March 2005.

[56] J. Haerri, F. Filali, C. Bonnet, "A Framework for Mobility Models Generation and its Application to Inter-Vehicular Networks", 3rd IEEE International Workshop on Mobility Management and Wireless Access (MobiWac'05), Maui, Hawaii, U.S.A., June 2005,

[57] The network simulator ns-2, http://www.isi.edu/nsnam/ns.

[58] Global Mobile Information Systems Simulation Library, http://pcl. cs.ucla.edu/projects/glomosim.

[59] QualNet Developer http://www.scalable-networks.com

[60] The OPNET Modeler, http://www.opnet.com/products/ modeler/home.html.

[61] F. Bai, N. Sadagopan, A. Helmy, "The IMPORTANT Framework for Analyzing the Impact of Mobility on Performance of Routing for Ad Hoc Networks", AdHoc Networks Journal - Elsevier Science, Vol. 1, Issue 4, pp. 383-403, November 2003.

[62] BonnMotion, http://web.informatik.uni-bonn.de/IV/ BonnMotion

[63] M.J. Feeley, N.C. Hutchinson, S. Ray, "Realistic mobility for mobile ad hoc network simulation", in Lecture Notes in Computer Science (LNCS), Vol. 3158, pp. 324-329, 2004.

[64] A. K. Saha, D. Johnson, "Modeling Mobility for Vehicular Ad Hoc Networks", Poster Session, 1st ACM Workshop on Vehicular Ad Hoc Networks (VANET 2004), October 2004.

[65] U.S. Census Bureau - Topologically Integrated Geographic Encoding and Referencing (TIGER) system, http: //www.census.gov/geo/www/ tiger. 
[66] Ertico, http://www.ertico.com/en/links/links/gdf_-_ geographic_data_files.htm

[67] ESRI Shape Files, http://www.esri.com/library/ whitepapers/pdfs/shapefile.pdf

[68] The Obstacle Mobility Model moment.cs.ucsb.edu/mobility/

[69] The SHIFT Traffi c Simulator, http: //path . berkeley • edu

[70] D. Choffnes, F. Bustamante, "An Integrated Mobility and Traffi c Model for Vehicular Wireless Networks", 2nd ACM Workshop on Vehicular Ad Hoc Networks (VANET 2005), September 2005.

[71] R. Mangharam, et al., "GrooveSim: a topography-accurate simulator for geographic routing in vehicular networks", 2nd ACM Workshop on Vehicular Ad Hoc Networks (VANET 2005), September 2005.

[72] CANU Project Home Page, http://canu.informatik. uni-stuttgart.de.

[73] Radio Propagation Modeling http://www.ipvs.uni-stuttgart. de/abteilungen/vs/abteilung/mitarbeiter/eigenes/ stepania/start/downloads

[74] S. Jaap, M. Bechler, L. Wolf, "Evaluation of Routing Protocols for Vehicular Ad Hoc Networks in City Traffi c Scenarios", in Proc. of the 5th International Conference on Intelligent Transportation Systems Telecommunications (ITST), June 2005.

[75] A. Mahajan, et al., "Evaluation of Mobility Models for Vehicular Ad-hoc Network Simulations", Technical Report N.051220, Florida State University, 2005.

[76] H. M. Zimmermann, I. Gruber, "A Voronoi-based Mobility Model for Urban Environments", in Proc. of the European Wireless 2005 (EW'05), April 2005.

[77] K. Konishi et. al, "MobiREAL Simulator - Evaluating MANET Applications in Real Environments", in Proc. of 13th IEEE International Symposium on Modeling, Analysis, and Simulation of Computer and Telecommunication Systems(MASCOTS2005), Atlanta, USA, 2005.

[78] ART?S: Advanced Runtime Infrastructure, http://pads.cs.unibo. it/dokuwiki/doku.php?id=pads : artis-doc

[79] GPS TrackMaker, http://www.gpstm.com

[80] A. Jardosh, E. Belding-Royer, et al., "Toward realistic mobility models for mobile ad hoc networks", in Proc. of the 9th Annual International Conference on Mobile Computing and Networking (MobiCom 2003), September 2003. 
[81] B. Zhou, K. Xu, M. Gerla "Group and Swarm Mobility Models for Ad Hoc Network Scenarios Using Virtual Tracks", in Proc. of IEEE MILCOM'04, 2004. 